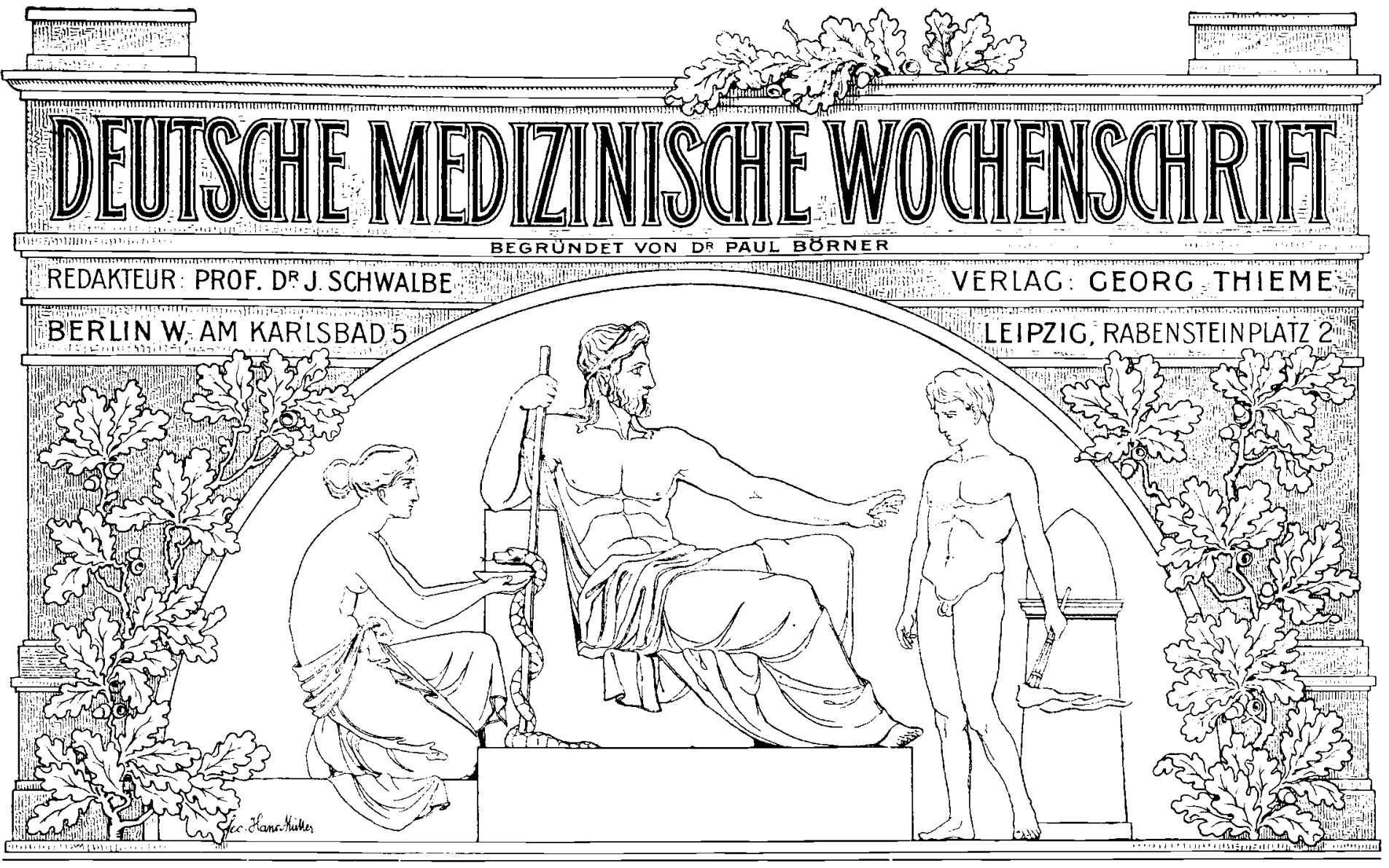

No. 43.

Donnerstag, den 26. Oktober 1905.

31. Jahrgang.

\section{Zur Diagnose, Prognose und Therapie der Paralysis agitans.}

Klinischer Vortrag.

\section{Von H. Oppenheim.}

M. H.: In einer vor wenigen Jahren erschienenen Studie welche Veister $\mathrm{Erb}{ }^{1}$, diesem Leiden gewidmet hat. findet sich unter den einleitenden Bemerkungen die. dilß wir jetzt. am Anfange des 20. Jahrhunderts, iiber ein vollkommen sicheres und fast erschöpfendes Syinptomenbild dieser Kranklieit verfügen. In seinen weiteren Ausführungen hält Erb das freilich nur für die typischen. wohl ausgebildeten Fälle dieses Leidens aufrecht. während er nicht verkennt und nicht verhehlt daß es Abarten und rudimentäre Formen gibt, deren Erhennung und Abgrenzung mehr oder weniger erhebliehe Sclswierigkeiten bereiten.

Obgleich ich diese Frage in meinem Lehrbuch der Nervenkrankheiten ziemlich eingehend behandelt habe. möchte ich ihr doch heute einige den Praktiker angehende Betrachtungen widmen.

Gewiß ist die Parkinsonsche Krankheit in ihrer typischen Form ein Leiden. das wie kaum ein anderes allf den ersten Blick, auf die Entfernung zu diagnostizieren ist. gewissermaßen seine ganze Symptomatologie anf der Oberfläche trägt. Ich darf auch voraussetzen, dab das charakteristische Bild ein jedem Arzt bekanntes ist und auf seine Schilderung verzichten. Aber meine Erfahrung hat mich gelehrt, dab die Anfangsstadien und die von dem Schultypus abweichenden Formen doch sehr häufig verkannt werden. In bezug auf diese dürften einige Winke und Aufklärungen nicht unwillkommen sein Aber mehr noch sind es die Schwierigkeiten in der Erkennung und Unterscheidung. die auch dem auf diesem Gebiete erfahreneren Arzte entgegentreten. welche mir den Anlab zu dieser Besprechung geben.

Daß es eine Paralysis agitans ohne Zittern gibt - diese schon 1875 von Charcot gelehrte Tatsache ist freilich so oft hervorgehoben worden, daß sie jedem Praktiker geläufig sein sollte. Um so mehr. als diese Form keineswegs eine seltene

1) Paralysis agitans (Parkinsonsche Krankheit). Die deutsche Klinik am Ein gange des 20. Jahrhunderts etc. Berlin-Wien 190 . ist. Erb berichtet, da\} von 183 Fällen seiner Praxis 37 . also etwa $90 \%$, in diese Klasse gehörten. Ich kann zwar nicht init Zahlen aufwarten. da ich über diesen Punkt in meinen Journalen nicht immer eine Notiz gemacht habe. aber nach meiner Schätzung ist der Prozentsatz der zu diesem Typus zählenden Fille eher ein noch gröilerer. Aber bei guter Ausbildung sind die durch die Muskelstare bedingten Haltungsanomalien und Deformitäten nebst der Beeinträchtigung (Verlangsamung) der aktiven Bewegungen doch so charakteristisch, daß die Liagnose leicht ist und nicht viel Gelegenheit zu Mibgriffen bietet.

Anders ist es mit den ersten Stadien und nit der so lıäufigen Begrenzung der lirankheitserscheinungen auf eine Körperseite. bzw. eine Extremität. Ich dart in dieser Hinsicht das zitieren. was ich in meinem Lehrbuch zu dieser Frage gesagt habe: "Eine in hüheren Alter sich langsam entwiekelnde Steifigkeit und Schwerfälligkeit in den Gliedmaß̧en einer Körper'seite ist meist Paralysis agitans, und gerade diese Form wird häufig verkannt."

Die Schwierigheit wächst nun erheblich, wenn eine objelitiv nachweisbare Juskelsteifigkeit nicht. bzw. noch nicht vorhanden ist. wenn der pathologische Zustand sich auf die bildsäulenartige Unbeweglichkeit einzelner Gliedmaßen (mit oder ohne Teilnahme einer oder beider Gesichtshälften sowie des Kopfes) beschränkt. aber bei der Funktionsprüfung weder eine Behinderung der Einzelbewegungen noch auch eine Erschwerung der passiven Bewegungen festzustellen ist. Es bleibt da nur der äußere Habitus - - insbesondere der Mangel der Ausdrucksbewegungen und der in der Norm vorhandenen Positionsveränderungen der Gliedmaßen --. welcher den Verdacht auf dieses Leiden hinlenkt. Gerade bei einseitiger Ausbildung ist diese Anomalie auch charakteristisch genug. unt die Diagnose zu ermöglichen.

Anders ist es jeduch, wenn sich diese Störung von vornherein generalisiert und in einer dem Greisenalter nahestehenden Periode zur Entwicklung kommt. Die Lebhaftigkeit des Mienenspiels und der Gestikulationen. die motorische Agilität ist ein individuell ungemein wechselnder Faktor und nimmt bekanntlich in höheren Alter mehr und mehr ab. I)a gibt es denn in der Tat Fälle, in denen es schwierig oder selbst unmöglich sein kann, festzustellen, ob der Zustand noch als ein physiologischer aufzufassen ist oder ob es sich um die 
beginnende Paralysis agitans handelt. Entscheidend für diese Beurteilung ist einmal der Umstand, daß eine wirklich erhebliche Veränderung gegen früher vorliegt und ferner, daß diese von dem Kranken selbst als ein belästigendes Novum empfunden wird.

Die diagnostische Bedeutung der Pro-und Retropulsion darf ich als bekannt voraussetzen und gleich auf einige, zum großen Teil von mir selbst beobachtete und an anderer Stelle beschriebene Zeichen hinweisen, die in derartigen zweifelhaften Fällen die Diagnose stützen können.

Eine in dieser Hinsicht besonders wertvolle Erscheinung ist die Verlangsamung der aktiven Bewegungen. So bekannt und so oft beschrieben das Symptom auch ist - eins wird doch nicht genügend beachtet und hervorgehoben, daß in den ersten Stadien und in der uns beschäftigenden Gruppe der atypischen Fälle sich diese Verlangsamung oft und ausschließlich und jedenfalls am deutlichsten an den distalen Teilen, besonders an den Fingern (und eventuell an den Zehen), zeigt. Der Patient, der den Arm schnell und vollkommen in der Schulter und im Ellbogengelenk bewegt, zaudert, wenn er die Finger beugen und strecken, ab- und adduzieren soll. Es geht das sehr langsam und unvollkommen vor sich, und besonders ist es mir aufgefallen, daß die Fähigkeit, einen Finger nach dem andern - jeden isoliert - schnell zu beugen und zu strecken, aufgehoben, bzw. stark beeinträchtigt ist.1)

Setzt die Affektion in einer der unteren Extremitäten ein oder wird gleichzeitig mit dem Arm das Bein in Mitleidenschaft gezogen, so macht sich dieselbe Anomalie im Bereiche der FuB- und Zehenmuskulatur geltend. Da aber die Beweglichkeit der Zehen großen individuellen Schwankungen unterworfen ist, so tritt die Erscheinung hier nur bei unilateraler Entwicklung des Leidens in überzeugender Deutlichkeit zutage.

Dabei ist mir dann noch eine Eigentümlichkeit aufgefallen, auf die durch eine Mitteilung meines Schülers D. Frank ${ }^{2}$ ) hingewiesen wurde: Versucht der Kranke die Zehen des von der Störung ergriffenen Beines zu bewegen, so stellen sich Mitbewegungen in den Zehen der gesunden Seite ein, während das umgekehrt nicht oder in weit geringerem Grade zutrifft. Ist das Symptom auch keineswegs ein konstantes, so hat es mir doch oft genug die Diagnose erleichtert.

Die Verlangsamung der Fingerbewegungen und die Einschränkung ihrer Exkursionen kommt auch meistens in der Schrift zum Ausdruck, sodai diese, auch da, wo das charakteristische Zittern fehlt, häufig verändert ist, und zwar ist es neben der Verlangsamung die Mikrographie, die oft schon frühzeitig die Natur des Leidens offenbart.

Bei dieser durch das Fehlen des Tremors ausgezeichneten Form gelingt es manchmal, durch eine aktive oder passive Stellungsveränderung derExtremität ein allerdings dann gewöhnlich nur schwaches und flüchtiges Zittern auszulösen. Fordert man z. B. den Kranken auf, die gebeugten Finger zu strecken und zu spreizen, so sieht man nicht selten ein Zittern in diesen, besonders im kleinen Finger, eintreten (meist in adduktorischer und abduktorischer Richtung). Diagnostisch ist es aber nur dann zu verwerten, wenn es die charakteristischen Eigentümlichkeiten des Parkinsonschen Zitterns darbietet. Von zweien meiner Patienten wurde angegeben, daß das Zittern nur beim Eintauchen der Hand in kaltes Wasser, resp. nachher eintrete - und der Versuch hat das bestätigt.

Bringt man den Fuß in dorsalflektierte Stellung und hält inn eine Weile in dieser, so werden die Fuß- und Zehenstrecker von einem rhythmischen Tremor befallen. Ich habe diese Erscheinung als "falsches FuBzittern " beschrieben, ${ }^{3}$ ) und wenn sie auch nur in einem Teil der Fälle deutlich nachzuweisen ist, kann sie doch bei zweifelhafter Diagnose zu einem brauchbaren Kriterium werden.

Es ist bekannt, daß rhe u ma toideSchmerzen zu den ersten Symptomen gehören können, doch erreicht diese Beschwerde nur ausnahmsweise einen hohen Grad. Vielfach bin ich auch

1) Ebenso ist es in den typischen, mit Tremor verknüpften Făllen zuweilen gerade dieser Akt, der gegen die Regel steigernd auf das Zittern wirken kann. 2) Zur Symptomatologie der Paralysis agitans. Monatschrift für Psychiatrie und Neurologie 1900, Bd. 8. - 3) Schon in der ersten Auflage meines Lehrbuches, und D. Frank ist in seiner Arbeit darauf zurūckgekommen. Compin hat das erstere in seiner These übersehen. der Angabe begegnet, daß Anfälle von Gelenkrheumatismus der Entwicklung des Leidens voraufgegangen seien. Es dürfte aber kaum zu einer allgemeinen Kenntnis gelangt sein, dab gastrische Störungen [Grawitz] meinen Erfahrungen ${ }^{2}$ ) die Salivation ein quälendes Frühsymptom bilden können. Vor kurzem sah ich einen Fall, in welchem der Entwicklung der typischen Symptome lange Zeit die Neigung zu gehäuften, dünnflüssigen Darmentleerungen - ganz ähnlich den Diarrhöen des Morbus Basedowii - vorausgegangen waren. Eine entsprechende Notiz findet sich auch in der These von Compin. ${ }^{3}$ ) Bei einem anderen meiner Patienten wurde der Zustand von Boas als Gastritis anacida bezeichnet. Mehrfach waren es einfache Cardialgien oder Darmkoliken, die im Vor- oder Frühstadium auftraten.

Es ist zwar die Regel, daß die Blasen- und Mastdarmfunktion unbeeinträchtigt bleibt; aber ich habe nun doch schon manchen sonst typischen Fall beobachtet, in dem eine nicht durch lokale Erkrankung (Prostatahypertrophie etc.) bedingte Pollakiurie, Dysurie, Incontinentia urinae, seltener alvi zu dem Symptomenkomplex gehörte. Es ist freilich entschieden ungewöhnlich, daß diese Störungen einen stabilen oder gar progredienten und prominenten Charakter haben.

Auf die typischen Gehstörungen brauche ich nicht einzugehen. Wenig beachtet sind aber bis jetzt die Gehhem m u gen, obgleich sich in den Arbeiten von Petrèn, bei einigen französischen Forschern, wie es scheint auch bei Knapp ${ }^{4}$ und in meinem Lehrbuch entsprechende Fingerzeige finden. Es gibt nämlich Fälle, in denen die Patienten beim Versuch vorwärtszuschreiten nicht nur die gewöhnliche Schwierigkeit des ersten Ansetzens zu überwinden haben, sondern lange Zeit wie angewurzelt dastehen oder beim Uebergang von einel Richtung in die andere, von einer Straße in das Haus, von einem Zimmer in ein anderes festkleben und wie ratlos dastehen. Zweifellos spielt hier ein psychisches Moment, ${ }^{5}$ ) eine Phobie, eine Rolle; hat sie auch ihre Quelle in der reellen körperlichen Bewegungshemmung, so bildet sie doch eirıen selbständigen sekundären Vorgang, und aus der Verflechtung dieser beiden Erscheinungen erwächst eine oft recht komplizierte, den Patienten sehr peinigende Funktionsbehinderung. Ich werde auf diesen Punkt bei der Therapie zurückkommen.

Gerade die atypischen Formen bedingen es, daß es eine große Reihe von Affektionen gibt, die dieser Krankheit ähnlich sehen oder ihr selbst so gleichen, daß die differentialdiagnostische Sonderung große Erfahrung und Sachkenntnis erheischt. Von den in dieser Hinsicht beachtenswerten Tatsachen dürfte den praktischen Arzt am meisten interessieren: die Vortäuschung. der Paralysis agitans durch Symptomenbilder der Hysterie, Hysteroneurasthenie und anderer Neurosen. Nach meiner persönlichen Erfahrung sind es fast ausschließlich Formen der traumatischen Hysterie, Hysteroneurasthenie oder Neurose, die der Paralysis agitans bis zur Verwechslung ähnlich werden können.

Einer traumatischen Form nichtechter Paralysis agitans bin ich zum ersten Male im Jahre 1888 begegnet und habe dann in den Charité-Annalen 1889, Jahrgang XIV, diesen Zustand unter dem Titel: Ueber eine der Paralysis agitans verwandte Form der traumatischen Neurose (Pseudo-Paralysis agitans) beschrieben.

Wenn ich heute, nach 17 Jahren, auf diese Frage zurückkomme, so geschieht es, weil mir die differentialdiagnostischen Schwierigkeiten trotz des enormen Fortschrittes in der Erkenntnis und Unterscheidung der Nervenkrankheiten auf diesem Gebiete eher größer als geringer geworden zu sein scheinen. Ich will der Betrachtung wieder einige Krankengeschichten, die ich im Auszug wiedergebe, vorausschicken.

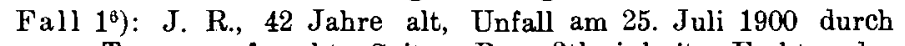
Sturz von Treppe auf rechte Seite. BewuBtlosigkeit. Fraktur des

1) Diese Wochenschrift 1894, No. 3. - 2) Zur Symptomatologie der Paralysis agitans. Journal für Psychologie und Neurologie 1902, Bd. 1, H. 4. - 3) Etude clinique des Formes anormales de la maladie de Parkinson. These de Lyon 1902. - 4) New York medical Journal 1891. - 5) Auf die anderweitigen psychischen Anomalien bei diesem Leiden und die sich gelegentlich mit ihm verknüpfenden Psychosen - eine Frage, welche von $B$ all, P aront, Roger, Wille $u$. a. behandelt worden Ist - will ich hier nicht eingehen. -6 ) Dieser Fall ist schon a. a. O. von meinem Assistenten Dr. G. Flatau unter dem Titel: Ueber einen Fall traumatischer Nervenerkrankung mit Paralysis agitans - ähnlichen Symptomen. Aerztliche Sachverstăndigenzeitung 1905,
No.4, veröffentlicht worden. 
rechten Armes. Suggillationen am rechten Beine. Schienenverband, nach drei Tagen Gipsverband für vier Wochen. Die dann bestehende Steifigkeit des rechten Armes wird mit mechanischen Bewegungen und Massage behandelt. Große Schmerzen. Schwellung des Handrückens. Frst nach einigen Monaten stellt sich Zittern im rechten Arme ein. das allmählich an Intensität zunimmt.

Status: Subjektiv: Zittern und Schwäche im rechten Arm. Schwäche und Parästhesien im rechten Oberschenkel, Kopfschmerz. Aufgeregtheit. Empfindlichkeit gegen Geräusch. Angst und Unsicherheit.

Gesichtsausdruck auffallend starr. Seltenheit des Lidschlags. Kopf passiv und aktiv frei beweglich. Andauernder Ruhetremor der rechten Hand. der sich in ganz gleiclimabiger, rhythmischer Weise in den Beugern und Streckern der Hand abspielt: besonders in den Extensores carp. rad. Hs kamen (nach meiner Erinnerung) etwa 4-5 Oszillationen auf die Sekunde. Durch aktive Bewegungen - Heben des Armes. Reichen der Hand etc. - wird das Zittern für einen Augenblick vollständig beseitigt. Kein Erzittern der einzelnen Finger, keine Pillendrehbewegungen.

Auch in der linken oberen Fxtremität ist das Zittern angedeutet. Durch aktive Bewegungen dieser Extremitait wird anch der Tremor der rechten Hand für einen Moment unterdrückt.

Händedruck rechts schwach.energielos. aber keine Verlangsamung der Fingerbewegungen. Die Schwäche (bzw. Anenergie) erstreckt sich auf die ganze rechte obere Extremität. Läßt man Patientin Beugung und Streckung im kllbogengelenk mehrere Male hintereinander ausführen. so läßt sich der Tremor der rechten Hand für geraume Zeit vollkommen unterdrücken. Nach einiger Zeit läßt sie dann den Arm wie einen olähmten herabfallen. und dann sistiert der Tremor für ungefähr zwei Minuten ganz. Ganz besonders wird das Zittern durch psychische Erregung gesteigert. Eine Hypertonie. bzw. eine Muskelspannung ist an keiner Stelle nachzuweisen. im Gegenteil: selbst an der am meisten betroffenen rechten oberen Extremität ist der Muskeltonus eher herabgesetzt. sodaß sich die Hand ohne jeden Widerstand passiv beugen und strecken läßt.

Die rechte Gesichtshälfte erscheint während der Untersuchung geschwollen. ihre Haut ist intensiv gerötet und fühlt sich wärmer an als die der linken Seite. An den Armen besteht kein Temperaturunterschied. Dagegen ist am ganzen rechten Bein die Haut kühler als links. Hyperästhesie in der ganzen rechten Körperseite. Knie- und Fersenphänomen beiderseits stark gesteigert. aber keine Rigidität. kein Babinski. kein dorsales Unterschenkelphänomen. Aktive Bewegungen im rechten Bein kraftlos. Durch Wachsuggestion läBt sich das Zittern nicht beeinflussen: auch in der Hypnose ist der Einfluß kein deutlicher.

Gang etwas langsam und schwerfällig. aber keine Pro- oder Retropulsion.

Patientin fixiert gewöhnlich die zitternde rechte Hand mit der linken.

Verlauf: Während der Beobachtungsizeit keine Besserung; indes Unfallansprüche.

Fall 2. A. Sch. 65 Jahre alt. Pferdebahnschaffner. Vor vier Jahren von einer Droschke überfahren. dabei Verletzung des rechten Armes und der rechten Gesichtshälfte. Bald nach Verheilung der Wunden stellte sich ein Zittern im rechten Arm ein. das sich all. mählich verstärkte. außerdem eine Bewegungsstörung im rechten Arm. besonders in der Schulter und Schmerzen. Er will seit jener Zeit ängstlich. aufgeregt und schreckhaft sein. Das Zittern ist ein andauerndes. nur für Minuten soll es zuweilen zurücktreten. Selbst aus dem Schlafe werde er öfters durch den Tremor geweckt.

Fis besteht eine Lähmung der linken Gesichtshälfte und eine linkseitige Ohraffektion. - Erscheinungen. die mit dem jetzigen Leiden in keiner Verbindung stehen.

In der rechten Hand besteht ein fortwährendes. sich in den Beugern und Streckern der Hand abspielendes. auffallend gleichmäßiges Zittern von vier bis sechs Schwingungen in der Sekunde. Die Finger sind in allen Gelenken gebeugt. doch nicht bis zum FaustschluB. Versucht man sie passiv aus dieser Stellung zu bringen. so macht sich ein starker. unüberwindlicher. scheinbar intentioneller Widerstand geltend. Sowohl bei aktiven als anch bei passiven Bewegungen nimmt das Zittern an Intensität zu. Fixiert man die Hand so greift der Tremor auf die Muskulatur des Oberarms und der Schulter über. Auf suggestivem Wege (verbal. Magnetapplikation) läßt sich die Erscheinung nicht beeinflussen.

Es besteht eine Hemihypalgesie an der ganzen rechten Körperseite, auch sind die sensorischen Funktionen, wenigstens Geruch und Sehvermögen (G F E) hier etwas beeinträchtigt.

Sehnenphänomene und Hautreflexe normal. Gang bietet nichts Besonderes. Tachycardie.
Bei einer sich über mehrere Jahre erstreckenden Beobachtung erfährt das Leiden keine Besserung.

Fall 3. J. F.. 49 Jahre alt. Arbeiter. For fünf Jahren geriet die rechte Hand in ein Schwungrad. es kam zu erheblicher Quetschung und Zerrung. deren Folgen monatelang bestehen blieben. Bald nachdem die chirurgische Behandlung abgeschlossen war. entwickelte sich ein Zittern in der rechten Hand. das allmählich stärker wurde und nach Jahresfrist auch auf das rechte Bein. dann in geringerem Maße auf den linken Arm iibergriff. Gleiclizeitig wurden die Bewegungen in diesen Körperteilen immer langsamer und schwerfälliger. desgleichen die Lokomotionen des ganzen Körpers Auch stellte sich Propulsion. seltener Retropulsion ein. ferner Hyperidrosis und Salivation.

Status: Typische Haltung des Kopfes. Rumpfes und der Gliedmaßen. Hände in Schreibstellung. Gesichtsausdruck starr. Selten heit des Lidschlags. Aber keine Muskelsteifheit. so läßt sich auch der Kopf passiv nach allen Richtungen frei bewegen.

Aktive Bewegungen in toto - - beim Aufstehen. Kehrt machen etc. - ebenso im einzelıen verlangsamt. wenigstens an den distalen Teilen. besonders den Fingern; Patient ist nicht imstande. einen Finger nach dem andern ansgiebig zu beugen und zu strecken.

Rhythmischer Ruhetremor der rechten Hand im Sinne der Pround Supination. Beugung und Streckung: auch die Finger zittern. und zwischen Daumen und Zeigefinger bestehen die charakteristischen Reibebewegungen. Aktive und passive Bewegungen wirken hemmend auf das Zittern. Schrift klein. zittrig.

Im rechten Bein besteht dieselbe Art des Zitterns. und zwar besonders in den Adduktoren sowie in den FuBstreckern. Keine Steifigkeit. Sehnenphänomene etwas erhöht. Reflexe normal. Falsches FuBzittern. Aktive Bewegungen an FuB und Zehen ver langsamt und eingeschränkt; beim Versuch. die Zehen des rechten Fußes zu bewegen. Mitbewegungen in den linken. nicht aber umgekehrt. Pro- und Retropulsion.

Im linken Arm geringes. inkonstantes. nicht typisches Zittern.

Hs besteht eine sich über die ganze rechte Körperseite erstreckende Hemihypalgesia mit Hyposmie. Hypageusie und konzentrischer Gesichtsfeldeinengung. besonders rechts. Patient klagt über Verstimmung. Reizbarkeit. Angst. Schmerzen im rechten Arm.

Verlauf: Unter wiederholter Applikation des Magneten schwindet die rechtseitige Hemianästhesie. Die übrigen Symptome bleiben unbeeinfluBt, erfahren im Lanfe von drei Jahren eine deutliche Steigerung. Auch Hyoscin hat keinen großen Effekt.

Das sich in diesen Fällen präsentierende Symptomenbild unterscheidet sich von dem der Paralysis agitans in einigen wichtigen Punkten. Es sind besonders: 1. das Fehlen der Muskelsteifigkeit; 2. das Vorhandensein von Krankheitssymptomen. die im Bilde der typischen Schüttellähmung zu fehlen pflegen: namentlich der Sensibilitäts- und sensorischen Störungen. der reizbaren Schwäche und der Angstzustände: 3. gewisse Eigentümlichkeiten im Verhalten des Zitterns etc.

Können diese Nomente als durchgreifende Unterscheidungsmerkmale betrachtet werden? Am meisten glaubte ich das für das Ausbleiben der Muskelstarre bei längerem Bestehen des Leidens annehmen zu müssen. Ich bin aber bezüglich der Bedeutung dieses Faktors im Laufe der Jahre unsicher geworden und habe jedenfalls Fälle gesehen. die bei im übrigen typischer Ausbildung des Symptomenkomplexes die Rigidität dauernd vermissen ließen. Ein solches Beispiel werde ich weiter unten beibringen. Erb hat offenbar dasselbe beobachtet. denn er wirft die Frage auf. ob es nicht auch eine Paralysis agitans ohne Steifheit der Muskelspannungen gebe. Ich muß freilich bekennen. daß meine entsprechenden Beobachtungen durch die enorme Langsamkeit des Verlaufs einen im ganzen gutartigen Charakter hatten. Weitere Erfahrungen könnten also dazu führen. hier einen besonderen Typus des Leidens abzugrenzen.

Das zweite Moment, das Hervortreten von Störungen der Sensibilität. sensorischen Störungen und anderen hysterischen oder neurasthenischen Erscheinungen. ist gewiß von nicht zu unterschätzendem Wert. Aber es bleibt doch zu beachten. daß die Paralysis agitans eine Krankheit ist. die sich mit andern Neurosen zu verknüpfen geneigt ist. Und namentlich liegt es nahe. daß die traumatische Aetiologie derartige Kombinationen hervorzubringen vermag.

So habe jch unter anderem auch einen Fall beobachtet. in dem eine Kreissä̈genverletzung der Hand neben einer Lähmung des Medianus und Ulnaris eine Paralysis agitans im Gefolge hatte. 
Es fragt sich also, ob wir in der Art und Beeinflußbarkeit des Zitterns entscheidende Anhaltspunkte finden. Dabei habe ich natürlich nur Fälle im Auge, in denen das Zittern in bezug auf Rhythmus, Frequenz und Lokalisation dem der Paralysis agitans sehr verwandt ist, muB aber zugestehen, daß diese Faktoren auch bei dem Leiden selbst gewissen Schwankungen unterworfen sind; am meisten gilt das für die Sichwingungsfrequenz, die ich zwischen drei und sieben Oszillationen (durchnittlich vier bis fünf) in der Sekunde schwanken sah. Immerhin läfit sich sagen, daß sich bei aller Aehnlichkeit doch auch meistens. Ilifferenzen finden, die eine Gegenüberstellung und Sonderung ermöglichen. So fällt es auf, daß bei diesen Pseudoformen die einzelnen Finger meist nicht für sich erzittern. lis braucht das freilich auch bei der echten Schüttellähmung nicht der Fall zu sein; wo es aber zutrifft, ist nach meinel Erfahrung Hysterie nicht im Spiele. Auch in dem Linfluß aktiver und passiver Bewegungen haben wir ein häufig ausschlaggebendes oder doch brauchbares hriterium. Bei der Parkinsonschen krankheit haben diese meistens eine beschwichtigende, tremorhemmende Wirkung, die soweit gehen kann. dab selbst langdauernde Arbeitsleistungen (Handarbeiten, Märsche etc.) ohne besondere Schwierigkeit ausgeführt werden können. In unseren Fällen ist diese Beziehung meist eine andere: In der Regel wirkt die aktive Muskeltätigkeit sch $n$ im ersten Moment erhöhend auf den Tremol oder es macht sich umgekehrt (wie in Fall 1) eine ungewöhnliche Beeinflussung in der Art geltend, daß auch in Anschluß an die Tätigkeit für längere Zeit das Zittern aufhört. Aber ess ist zuzugeben, daß auch bei der echten Paralysis agitans, namentlich in den späteren Stadien, die aktive Bewegung einen tremorsteigernden Effekt haben kann.

Der Einfluß der passiven Bewegungen ist, obgleich er von mir wiederholentlich geschildert worden ist, merkwürdig wenig beachtet worden auch bei Erb findet sich nicht ein Wort darüber.) Und doch glaube ich. ihm in differentialdiagnostischer Hinsicht einen Wert zuschreiben zu dürfen. Bei der echten Schüttellähmung läßt sich nach meiner Erfahrung fast ausnahmslos der beschwichtigende Einflub variierter passiver Bewegungen feststellen, während sich bei der Pseudoform meist schon beim Versuch das Zittern lebhaft steigert

Man sollte nun meinen, dab wir in dem Verhalten des Tremors gegenüber den psychischen Reizen die sicherste Handhabe für die Differentialdiagnose hätten. Aber das trifft nicht ohne weiteres zu, und gerade das ist von Krafft-Ebing u. a. übersehen worden. Denn es gilt auch für die echte Paralysis agitans, daß 1. psychische Erregungen das Zittern lebhaft steigern; 2. Ablenkung der Aufmerksamkeit eine momentan hemmende Wirkung entfaltet; 3 . gewisse Störungen, wie die Abasie etc, durch psychischen Ansporn gelegentlich iiberwunden werden können.

Da indes die Kardinalsymptome dieses Leidens keineswegs einen psychogenen Ursprung haben, besitzen wir in dem Nachweis dieses Charakters (in der Feststellung des psychogenen Lrsprungs) der Erscheinungen die sicherste Unterlage für die Deutung des Leidens.

Wenn wir die angeführten Momente als Prüfstein für die oben geschilderten Fälle verwerten, so glaube ich, den elsten und zweiten unter die Rubrik der PseudoParalysis agitans, d. h. einer nicht mit der Schüttellähmung identischen Neurose, bringen zu müssen, ohne daß ich den ersten als Hysterie deute, während ich in dem dritten eine Kombination der echten Schüttellähmung mit Hysterie diagnostiziere. ${ }^{1}$ )

Daß das Vorhandensein der echten Pro- und Retropulsion, der Salivation, der charakteristischen Sprach- und Stimmstörung etc. zugunsten der Paralysis agitans spricht, bedarf kaum der Hervorhebung. So wird sich wohl meist eine sichere Entscheidung treffen lassen, aber die Schwierigkeiten der Entwirrung können recht bedeutende sein. Und selbst die längere Beobachtung und die Art des Verlaufs führt nicht immer zu einem sicheren Urteil, sodab es mir scheint, als ob es außer den hysterischen Afterformen noch anderweitige Neurosen

1) Derartige Kombinationen sind auch von $B$ échet (Thèse d. Paris 1892) und Chabert (Arch. d. Neurol. 1893) beschrieben worden. gäbe, die der Paralysis agitans weit näher stehen als der Hysterie. Wenn mich nicht alles täuscht, ist auch Erb geneigt, das nach seinen Erfahrungen anzunehmen.

Auf andere diagnostische Schwierigkeiten, die aus der Verknüpfung der Paralysis agitans mit Arteriosklerose und deren Folgezuständen - so findet sich die Verbindung des Leidens mit vitium cordis häufig bei meinem Material -. mit chronischem Gelenkrheumatismus, aus den merkwürdigen Mischformen mit Tabes, Sclerosis multiplex, mit der senilen Degeneration etc. resultieren, will ich hier nicht eingehen, sondern mich nun der bedeutsamen Frage nach der Prognose und Therapie zuwenden.

Ueber die Prognose der Paralysis agitans herrscht unter den Kennern dieses Leidens kaum eine Meinungsverschiedenheit. Alle bezeichnen sie als traurig, trostlos, wenn sie auch zugestehen, daß es schwere und weniger schwere, rascher und langsamer verlaufende Fälle gibt und daß gelegentlich Remissionen ${ }^{1}$ ) vorkommen. Ich selbst hatte in dieser Hinsicht zunächst die sehr pessimistischen Anschauungen meines Lehrers Westphal überkommen, in denen ich durch die Beobachtungen. welche ich in der Charité anzustellen Gelegenheit hatte, bestärkt wurde. Diese Auffassung ist auch noch in den ersten Auflagen meines Lehrbuchs zur Geltung gekommen, und nichts ist fïr sie bezeichnender, als daß das Kapitel Therapie hier mit dem Satze eingeleitet wild: „Ler Arzt kann bei dieser Krankheit viel schaden und wenig nützen."

Mit der Erweiterung meiner Erfahrungen und besonders mit der Ausdehnung meiner nichtpoliklinischen Praxis hat sich meine Ansicht zwar nicht von Grund aus umgestaltet. aber doch eine wesentliche Modifikation erfahren. Und besonders der Wunsch, das zu erläutern und in einem von den praktischen Aerzten gelesenen Journal zu betonen, hat mir den Anstob zu dieser Publikation gegeben. Freilich kenne ich auch heute keinen Fall von Heilung der Paralysis agitans. Aber ich habe eine ziemlich große Zahl von Fällen gesehen, in welchen die Krankheit hinsichtlich der Intensität ihrer Beschwerden und der Langsamkeit ihres Verlaufs als eine gutartige bezeichnet werden mußte: ferner eine Reihe anderer, in denen es durch die Therapie gelang, den schweren, überaus qualvollen Zustand in einen milderen zu verwandeln. Ich will einige Beispiele dafür in aller Kürze anführen:

Fall 1. A. L., Kaufmann, 61 Jahre alt. Beginn 1891 mit Zittern im rechten Arm. 1893 typischer Tremor, Muskelsteifigkeit, Haltungsanomalie an diesem Arm ohne sonstige Erscheinungen.

Zweite Untersuchung 1896. Status idem.

Dritte Untersuchung 1899. Zittern im rechten Arm etwas heftiger. Patient geht aber noch wie früher seinem Berufe nach und wird durch das Zittern nicht wesentlich gestört.

Vierte Untersuchung 1903. Zittern rechts wie früher; auch die linke Hand zittert etwas. Leicht gebückte Haltung. Subjektiv relatives Wohlbefinden. Arbeitsfähigkeit nicht wesentlich vermindert.

Fall 2. R. J., Rentier, 58 Jahre alt. Erste Untersuchung 1896. Seit zwei Jahren Tremor im rechten Arm und Bein und Verlangsamung der Bewegungen. Objektiv in diesen Gliedmaßen typischer Befund.

1900. Status idem.

1904. An den Gliedmaßen derselbe Befund. Leichte Neigung des Rumpfes nach vorn. Geringe Nackenstarre. Patient macht lange Spaziergänge, spielt Karten, Billard, fühlt sich im Lebensgenuß nicht wesentlich behindert.

Fall 3. Frau Z., 50 Jahre alt. Erste Untersuchung im April 1894. Seit einigen Monaten hat sie große Mühe, sich beim An- und Auskleiden der linken Hand zu bedienen, auch will sie nicht so schnell rom Fleck kommen beim Gehen etc. Typisches Bild der Paralysis agitans sine agitatione. Muskelsteifigkeit und Bewegungshemmung, vorwiegend in den linkseitigen Gliedmaßen.

Letzte Untersuchung im Jahre 1902 . Zustand gegen früher nicht wesentlich verschlechtert.

Fall 4. L., Fabrikbesitzer aus Holland, 55 Jahre alt. Seit fün bis sechs Jahren Zittern in beiden Armen, Steifigkeit in den Beinen, Propulsion, zunehmende Gehstörung. Im Laufe des letzten Jahres: Kräfteverfall, Schlaflosigkeit, Gehunfähigkeit.

St a tu s: Vorzeitig gealterter Mann, zusammengekauerte Haltung. stärkste Ausbildung der charakteristischen Symptome der Paralysis

1) Am bcstinmtesten finde ich das bei Wollenberg (Paralysis agitans, Noth n age 1s Handbuch Bd. 12 , H. 2, 1899) betont. 
agitans. Das Zittern betrifft alle vier Extremitäten und die Lippenmuskeln. Muß mit fremder Hilfe vom Stuhle gehoben werden, geht nur mit doppelseitiger Unterstiitzung nach langem Zögern und ist nach wenigen Schritten wieder nicht vom Fleck zu bringen etc.

Therapie: Subcutane Hyoscininjektionen, elektrische Vierzellenbäder, passive Bewegungen der Gliedmaß̉en, Gehübungen etc.

Im Verlauf von drei bis vier Monaten ist der Zustand soweit gebessert, daß der wie verjüngt aussehende Mann allein große Spaziergänge macht, gut schläft und nur bei Aufregungen ein Zittern verspürt. Immerhin sind die Grunderscheinungen der Schüttellähmung in schwacher Ausbildung vorhanden. Hyoscin wird noch täglich in einmaliger Dosis von 0,0002 gebraucht. Gewichtszunahme zwölf Pfund.

Fall 5. Dr. S., Arzt, 66 Jahre alt. Krankheitsbild dem unter Fall 4 beschriebenen sehr ähnlich, auch in bezug auf die Intensität, doch ist die erhebliche Verschlimmerung erst in den letzten Monaten eingetreten: Patient hat sechs Wochen Tag und Nacht im Lehnstuhl zugebracht, weil beim Liegen das Schüttelı unerträglich w'urde. Schlaf sehr schlecht ete.

Behandlung wie im obigen Falle mit demselben bedeutenden Erfolge.

Fall 6. A. J., 60 Jahre alt. Torgeschrittene Paralysis agitans mit starker Ausbildung der Kontraktur und des Tremors. Heftige Angstzustände, insbesondere Basophobie. Wesentliche Besserung, besonders der letzterwähnten Beschwerden durch Psychotherapie, Gymnastik und Gehübungen

Ich könnte eine Reihe ähnlicher Beobachtungen mitteilen, möchte aber das Material hier nicht unnötig häufen. Nur ein Fall, der ein hervorragendes Interesse hat, verdient hier noch angeführt $\mathrm{zu}$ werden. $\mathrm{Er}$ ist im Jahre 1879 voll Westphal') beschrieben worden.

Das Leiden hatte sich bei dem 48 jährigen Manne vor 1.5 Jahren entwickelt Das Zittern war schon dauls stark ausgebildet und betraf Kopf, Unterlippe, Kiefer und obere Extremitätell. Der Charakter des Tremors war ein durchaus typischer; ungew ïhnlich war nur die Kopfhaltung, indem dieser nicht nach vorn. sondern nach hinten genejgt gehalten wurde; die Stellung der Extremitäten und des Rumpfes entsprach aber wieder der typischen.

Diesen Kranken habe ich nun unter Augen behalten, bin ihul oft auf der Straße begegnet und habe ilın vor einigen Wochen wieder untersucht. Er ist 77 Jahre alt, sein Leiden besteht seit etwa 40 Jahren, und wenn sich auch die Intensität des Tremors gesteigert hat und die Kyphose eine beträchtlichere geworden ist, so ist Patient doch auch heute noch durchaus mobil und leidet nicht viel mehr als vor 30 Jahreu.

Vun kann man gewiß einwenden, daß der Fall nur in bezug auf den Tremor und die Haltungsanomalien (auch der Kopf hat jetzt eine mehr geneigte Haltung) ${ }^{2}$ ) ein typischer ist. während das relativ frühe Einsetzen und der definitive Mangel der Kontraktur ihm eine gewisse Sonderstellung gibt. Aber das ist es ja gerade, was bewiesen werden sollte: daß es eine Paralysis agitans ohne Muskelsteifigkeit gibt, die, wie wir nun hinzufügen können, sich über Jahre und Dezennien erstrecken kann, ohne eine wesentliche Progression zu erfahren.

Ich habe auch einigemale Individuen behandelt, die mich wegen eines andern Leidens konsultierten und ihr seit langem bestehendes Parkinson sches Zittern so wenig störend empfanden, daß es für sie gar nicht die Bedeutung eines Symptomes hatte.

Ich möchte aber nicht den Eindruck erwecken, als ob ich nun zu einem unberechtigten Optimismus hinneigte, und will deshalb gleich ergänzend anführen, daß ich auch noch in den letzten Jahren eine Reihe von Personen an diesem Leiden behandelt habe, bei denen jede Therapie fehlschlug, die Affektion unaufhaltsam vorschritt und der Zustand etwa nach fünfbis zehnjähriger Dauer ein qualvoller wurde.

Ganz im allgemeinen kann man sagen, daß die Lebens. dauer durch diese Krankheit kaum abgekürzt wird, ja ich habe den Eindruck gewonnen, daß sie bei Personen auftritt, die meist aus langlebigen Familien stammen und auch selbst ein relativ hohes Alter erreichen.

Vieles, was sich auf die Behandlung des Leidens bezieht, ist schon im Laufe dieser Betrachtung erwähnt worden,

1) Zur Paralysis agitans. Charité-Annalen, IV. Jahrgang.

2) DaB der Haltungstypus im Laufe der Zeit derartige Wandlungen erfahren kann wird auch von Bid on (Revue de Méd. 1901) angeführt. doch will ich der Therapie einige besonclere Bemerkungen widmen

Der ungünstige Einflub der psychischen Erregungen hat zu der Empfehlung geführt, den Kranken bis zu einem gewissen Grade $z u$ isolieren, aus dem Getriebe del Geselligrlieit zu entfernen etc. - ich habe mich aucli selbst in diesen sinne ausgesprochen. Aber hier ist doch ein Variieren. eine Berücksichtigung der individuellen Neigungen und Verlialtnisse selı erfordellich. Es gibt Kranke dieser Art, die sicll im Verkehr mit anderen sehr wohl fühlen, an der Cnterhaltung, am spiel etc. mit Vergnügen und subjektivem Wollbefinden teilnehmen Einer meiner Patienten spielt stundenlang Billard, ein illuderer Schach, ohne davon einen Nachteil zu haben, ein dritter weht in seinen Club und vergibt dort sein Leiden. Lintel solehen Verhältnissen wäre eine lsolierung rigoros und hätte entsetieden eher einen nachteiligen Kinfluß. Die Behandlung von Fall zu Fall ist also das in dieser Hinsicht Empfehlenswerte.

In bezug auf die physikalischen Heilnethoden gilt auch heute, daß jede eingreifende fiur zu vermeiden ist. Von der Hydrotherapie habe ich nur dann Vorteil, und zwal auch nur bescheidene Erfolge gesehen, wenn sie sich auf eine einmalige, milde Applikation des Wassers (pro Tag) beschränite. Von kalten oder heilßen Vollbädern, Seebiidern und von Duschen ist iringend abzuraten, besonders aber von einer Häufung derartiger Prozeduren, während eine küłlle Waschung. ein laues Vollbad oder Halbbad, eine partielle Abreibung dem Krankerl dienlich sein kann.

Von tler Balneotherapie habe ich nicht viel Erfreuliches gesehen, doch ist z. B. Erb für die kühlen indifferenten Thermen von Johannisbad, Schlangenbad, Willbad etc. eingetreten. aber seine Empfehlung klingt auckl eher kühl-indifferent als begeistert. - Luftkuren spielen zwar keine herworagende Rolle, zweifellos hat aber del Aufenthalt im waldigen Mittelgebirge meist einen wohltuenden Einfluf

Die Körperinassage kann ich nach meinen Erfallurulgen nicht empfehlen, obgleich hiel und da einmal ron eincl günstigen Wirkung berichtet wird.

Dagegen habe ich schon friihel (z. B. 1. Aufl. meines Lehrbuches auf ein Verfahren hingewiesen, dessen lindernde II irhsamkeit ich immer wieder rühmen mub: die Anwendung pas. siver Bewegungen. Sie haben einen doppelten oder selbst dreifachen Zweck: 1. kann man durch zweckmälijg variierte, sanfte, passive Bewegungen der zitternden Teile den Tremor für die Dauer der Sitzung hemmen: ¿.. wird durch delartige Manipulationen der Entstehung und Progredienz der Steifigkeit bis zu einem gewissen Grade entgegengearbeitet; 3 . sind die Kranken in den späteren Stadien, in denen sie selbst die Position der einzelnen hörperteile und des ganzen hörpers nicht zu ändern vermögen, auf eine derartige Hilfeleistung angewiesen. Ich lasse diese passive Gymnastik mehrmals am Tage für die Dauer von fünf bis zehn Minuten ausführen -- immelaber dabei dem subjektiven Empfinden Rechnung tragend.

Gegen die aktive Gymnastik, die von Taylor, Fried. länder u. a. gerühnt wird, habe ich mich früher ablehnend verhalten und kann ihre Anwendung auch heute nicht generel befürworten. Aber ich habe doch auch einzelne Fälle gesehen, in denen das Verfahren, mit Vorsicht ausgeübt, sich nutzbringend erwies.

So erinnere ich mich an einen alten Herrn, der an einer uncollkommen entwickelten Form der P. a. litt und immer wieder $\%$ der Gymnastik zurückgriff, weil er die Ueberzeugung hatte. (lil) sie seine Extremitat beweglicher machte. In einem andern schweren, vorreschrittenen Falle hatten die gymnastischen Iebungen mehr die Bedeutung eines die Psychotherapie unterstiitzenden mid vermittelnden Faktors. Es gelang auf diese Weise. den l'atientin. der fast schon auf den Rollstuhl angewiesen zu sein schien. bei den aber das Nichtoehen zum Teil phobischen Ursprungs war. wieder weit beweglicher za machon. wieder auf die Beine zu bringen.

Der Wert der Gymnastik ist also nicht zu unterschätzen, doch heißt es hier wie überall, das Verfallen dem einzelnen Fall anzupassen, den individuellen Erfolg als Richtschnur zu benutzen und sich vor jedem Zwang, vor jeder Uebertreibung zu hüten.

Die Elektrizität in Form der lokalen Faradisation und Galvanisation hat mich immer im Stich gelassen, während 
Erb die Anwendung des konstanten Stromes - an Kopf, Nacken, Sympathicus etc. - gutheibt. Anders ist es mit dem Heilwert der elektrischen Bäder. Schon in der ersten Auflage meines Lehrbuchs (1894) berief ich mich auf zwei Fälle eigener Beobachtung, in denen diese Behandlung (wer sie zuerst empfohlen hat, ist mir nicht bekannt) einen gewissen Nutzen hatte. Seit jener Zeit habe ich den Kreis meiner Erfahrungen wesentlich erweitert und darf sagen, dab in einem nicht geringen Prozentsatz der so behandelten Fälle der Erfolg ein befriedigender und einigemale selbst ein ausgezeichneter war. Und zwar gilt das sowohl für die bipolaren faradischen, als auch für die elektrischen Vier. zellenbäder. Meist verringerte sich die Wirksamkeit allmählich und bei späterer Wiederaufnahme der Kur. Oft versagte sie ganz, ausnahmsweise wurde über einen entschieden ungünstigen Einfluß geklagt.

Die Nervendehnung und die Suspension darf als endgültig abgelehnt gelten.

Einzelne dieser Patienten machen die Bemerkung, daß sie sich bei Eisenbahnfahrten wohler fühlen. Diese Erfahrung hatte bekanntlich Charcot veranlabt, die Vibrationstherapie, insbesondere den Fauteuil trépidant zu empfehlen, ich kann jedoch nicht sagen, daß die Vibrationstherapie in irgend einer Form sich mir bei dieser Krankheit bewährt hätte.

Die medikamentöse Behandlung ist es nun, die in symptomatischer Hinsicht bei diesem Leiden am meisten leistet. Von den verschiedenen Tonica und Nervina wird bald dieses, bald jenes Präparat subjektiv wohltuend empfunden. Am meisten Anwendung finden im allgemeinen die Brompräparate und Arsenik. Erb verordnet die Solut. Fowler., Aq. Foenicul. und $\mathrm{Tt}$. nuc. vomic. zu gleichen Teilen, davon dreimal täglich 6-15 Tropfen. Ich pflege die Tt. Gelsemii sempervirentis, zuweilen auch die $\mathrm{Tt}$. Veratri viridis in kleinen Dosen $\mathrm{zu}$ verordnen, ohne dab ich diesem Mittel mit der Ueberzeugungskraft des Ueberzeugten das Wort rdeen kann.

Bei der Anwendung von Abführmitteln, und besonders bei der von Diaphoretica ist eine gewisse Vorsicht am Platze, da ich es zweimal erlebt habe, daß das Symptom der Hyperidrosis auf diesem Wege ausgelöst wurde.

Zweifellos hat sich Erb durch die Einführung des Hyoscin in die Therapie der Paralysis agitans ein großes Verdienst erworben.

Und das dann von Men del empfohlene Duboisin hat sich ebenfalls als ein brauchbares Mittel erwiesen. Ich selbst habe mich anfangs - vielleicht stand mir kein reines Präparat zu Gebote - durch die toxischen Wirkungen dieser Stoffe abschrecken lassen, habe abel schon in der zweiten Auflage meines Lehrbuches von Erfolgen gesprochen - der Vorwurf, den mir Erb in dieser Hinsicht jüngst gemacht hat, zeugt also dafür, daß er die Entwicklung meiner Anschauungen nicht weiter verfolgt hat - und kann nun auf Grund ausgedehnter Erfahrung als Lobredner dieser Therapie auftreten. Freilich liißt sie mich auch heute noch oft im Stich, wird sie von vielen Kranken nicht vertragen, aber die Zahl der Fälle, in denen sie sich wilksam zeigt, ist doch keine geringe. Wichtig ist es, daß man sich eines guten Präparates und möglichst frisch bereiteter Lösungen bedient, diese vor Zersetzung schützt, die Spritze sehr rein hält, und mit der minimalen Dosis von 0,0001 des Scopolamin. hydrobromicum beginnend, durch allmähliche Steigerung um je $1 / 2$ Dezimilligramm die Dosis ausfindig macht, die sich gerade wirksam zeigt, ohne wesentliche Intoxikationssymptome zu erzeugen. Wir sind meist mit zwei bis drei Dezimilligramm und einmaliger Tagesdosis ausgekommen. Wichtig ist die Tatsache - und auch darin kann ich Erb und Hilbert zustimmen - - daß das Mittel lange Zeit (ein Jahr und darüber) verabreicht werden kann, ohne daß sich sein Einfluß abzuschwächen braucht. Die interne Anwendung ist im ganzen weniger wirksam, lieB sich aber doch in einigen meiner Fälle durch Monate mit Nutzen durchführen. Hilbert gibt den Rat, das Medikament nur jeden zweiten bis dritten Tag subcutan, in der Zwischenzeit innerlich zu geben.

Bei dem einen Kranken hat das Hyoscin, bei dem andern das Duboisin größeren Effekt und wird besser vertragen, doch verdient das erstere im ganzen den Vorzug und ist in kleinerer
Dosis wirksam, während vom Duboisin durchschnittlich zwei bis vier Dezimilligramm mehrmals am Tage erforderlich sind. Der palliative Einfluß dieser Mittel erstreckt sich in erster Linie auf das Zittern, dann auch auf die Muskelsteifigkeit, schließlich wird durch das Nachlassen der letzteren auch die aktive Beweglichkeit mehr oder weniger erhöht. Ueber die Rhizoma Scopoliae carniolae $(0,2-0,4)$, die Podak empfahl, habe ich zu wenig eigene Erfahrung.

Daß wir in den Endstadien und schwersten Fällen nicht ohne das Morphium auskommen, bedarf kaum der Erwähnung. Gegen die oft recht quälende Salivation habe ich einigemale Gurgelungen mit einigen Tropfen Tinct. jodi in einem Weinglase Rotwein mit Erfolg angewandt.

So befremdend es auch zunächst klingen mag - die Psychotherapie hat auch bei diesem Leiden ein Feld der Betätigung. Nicht etwa nur in dem Sinne, daß es für jeden unheilbar Kranken von großem Wert ist, wenn sein Mut belebt, seine Hoffnung aufrecht erhalten, sein Geist von dem Leiden abgelenkt wird. Nein, es gibt hier auch spezielle und weit bestimmtere Indikationen. Soweit meine Erfahrung reicht, sind es Symptomenkomplexe, die aus dem Leiden herauswachsen, bzw. sich ihm aufpfropfen, besonders die schon angeführten Hemmungen und Phobien, welche durch psychischen Ansporn und Psychogymnastik gebessert und überwunden werden können.

Ich habe mich davon mehrfach überzeugt und auch schon oben beiläufig áuf die Tatsache hingewiesen. Die Hypnose habe ich nicht selbst angewandt, aber ein von Gumpert erzjelter Erfolg läßt doch erkennen, daß man in einzelnen Fällen auch dieses Heilverfahren mit Nutzen in Anwendung ziehen kann. Die auf Grund theoretischer Erwägungen (Möbius, Lund. borg) versuchsweise angewandte Organotherapie, die Darreichung ven Thyreoidin und anderen Organextrakten (Dana) hat zu negativen Ergebnissen geführt. -

So erfreulich also die hier verzeichneten Fortschritte in der Behandlung sind, von dem erstrebenswerten Ziele, dieses Leiden zur Heilung führen zu können, sind wir noch weit entfernt. 